\title{
REASONS OF WORKFORCE EMIGRATION FROM GEORGIA TO EUROPE AND EMPLOYMENT PROBLEMS
}

\section{KESO SUMBADZE}

PhD in business administration, Invited Assistant Professor

Business and Technologies University, Georgia

Keso.sumbadze@btu.edu.ge

Abstract. If we consider unemployment level in Georgia and Europe, multitude of problems, that is visible in this regard, won't be hard to be detected. Finding a job is a vitally important issue for a person. However, by looking at current job openings in labor market, requirements complexity and unreality will be noticed in fact. Besides, there are several problems that studying and elimination are essential for both job-seekers and employed people.

Exited severe social-economic background, high level of unemployment and unfavorable employment conditions in Georgia, promote workforce migration. The closest job market for our country's citizens is European countries, where they have to deal with too complicated situations.

The purpose to this article is to draw attention for Georgia and European countries' topical issues such as: Unemployment, jobs search and difficulties arisen it that process, workforce emigration caused by high level of unemployment, in Europe and came across employment problems. Problems, revealed as research results, and developed recommendation to solve them will be suggested in detail.

\section{KEYWORDS: UNEMPLOYMENT, EMIGRATION AND EMPLOYMENT PROBLEMS IN EUROPE.}

For citation: Sumbadze, K. (2020). Reasons of workforce emigration from Georgia to Europe and employment problems. Globalization and Business, №9, pp. 86-90. (In Georgian) https://doi.org/10.35945/gb.2020.09.010 


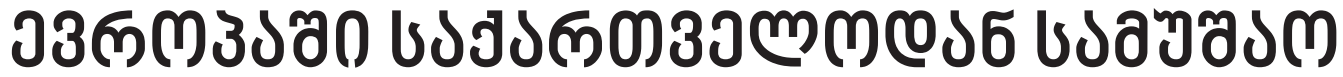

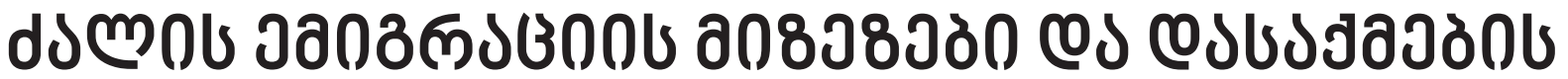

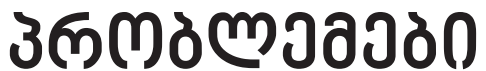

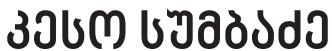

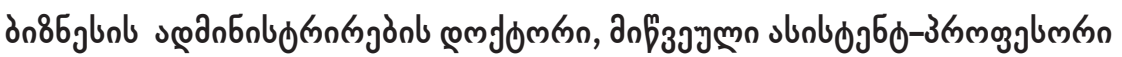

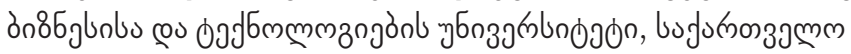

Keso.sumbadze@btu.edu.ge

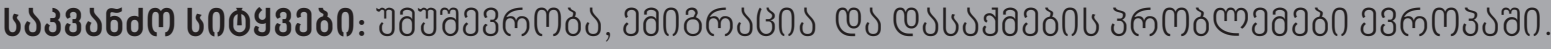

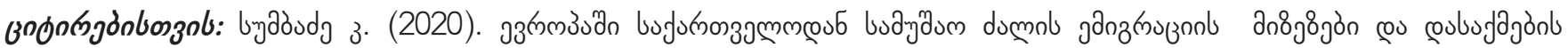

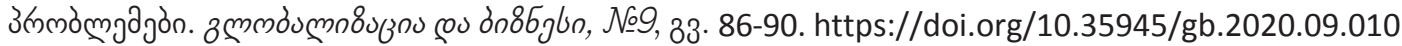

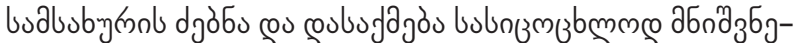

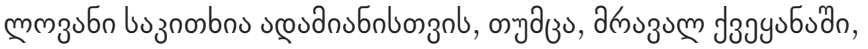

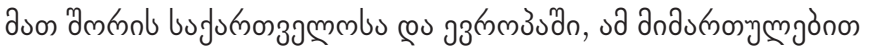

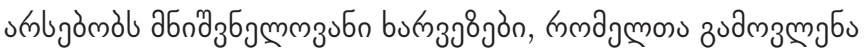

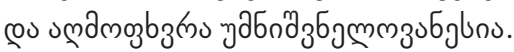

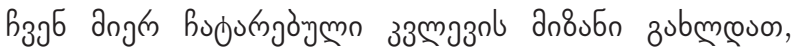

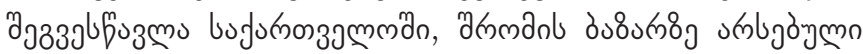

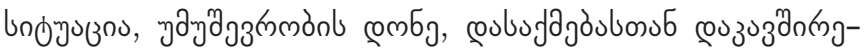

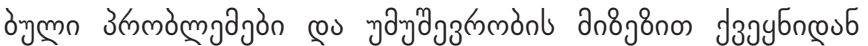

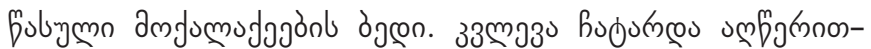

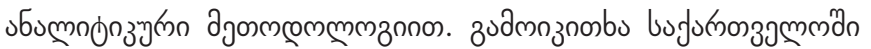

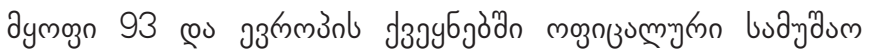

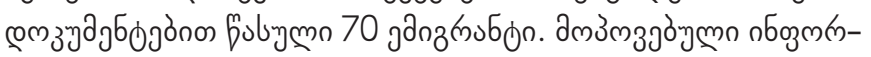

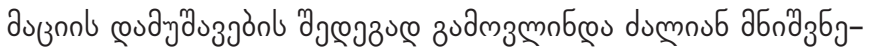

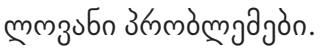

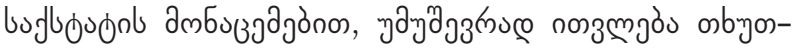

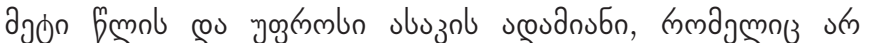

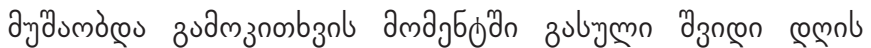

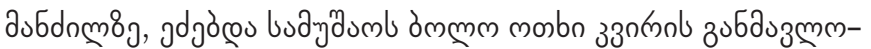

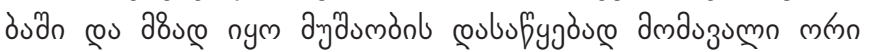

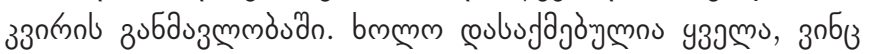

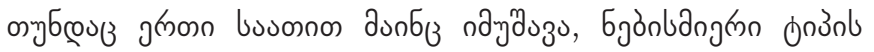

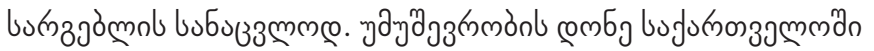

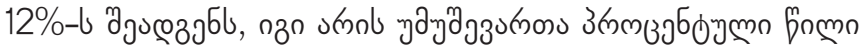

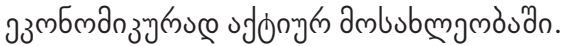

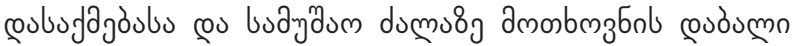

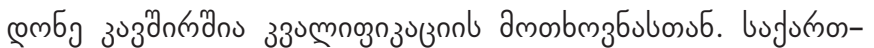
зэmm

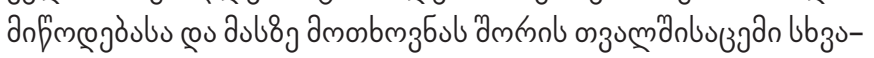

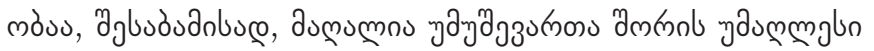

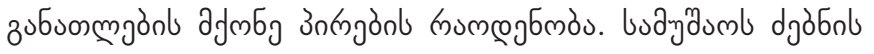

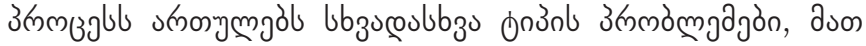

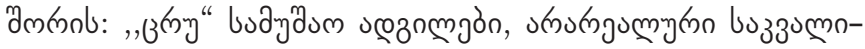

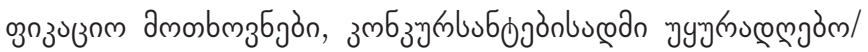

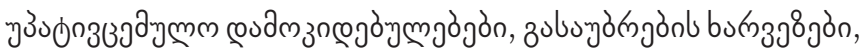

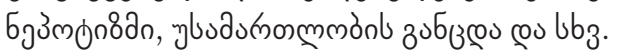

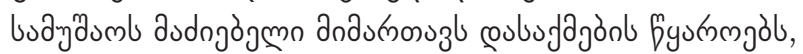

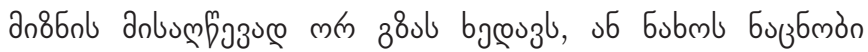

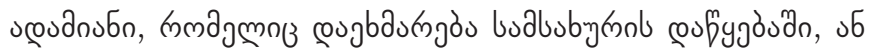

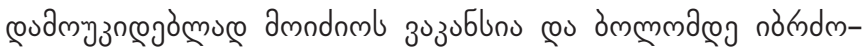

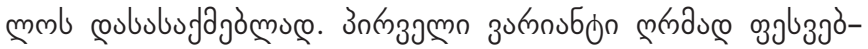

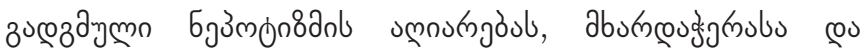

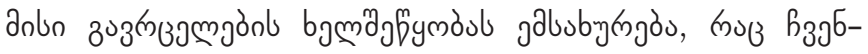

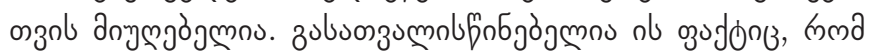

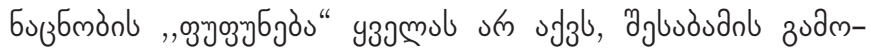

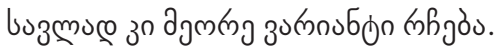

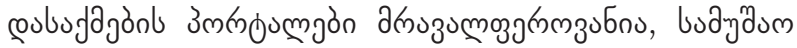

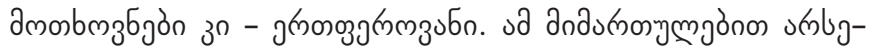

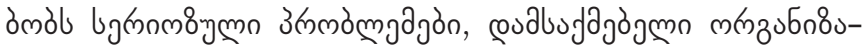

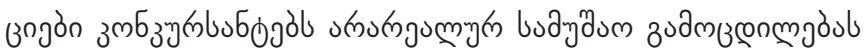

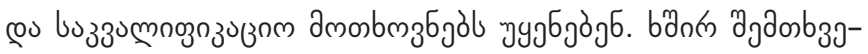

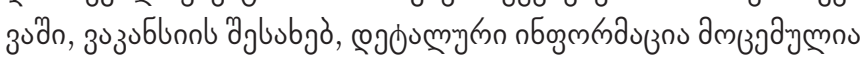

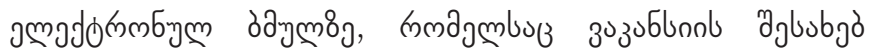

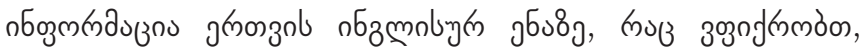

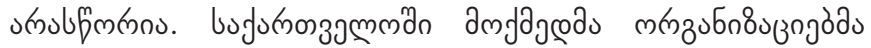

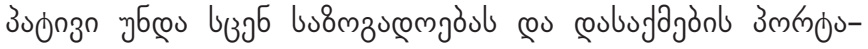

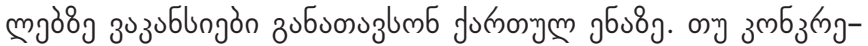

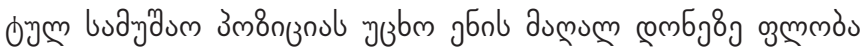

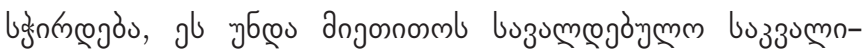

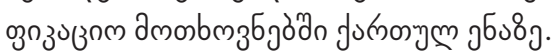

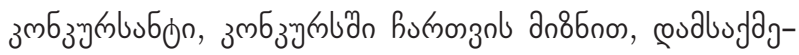

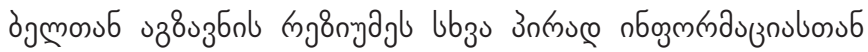




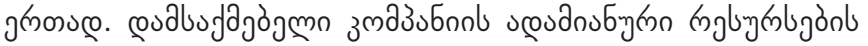

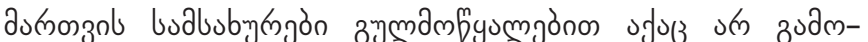

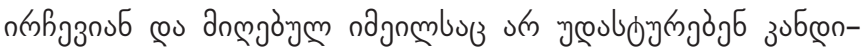

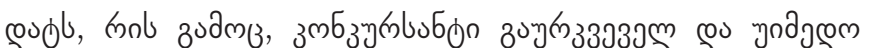

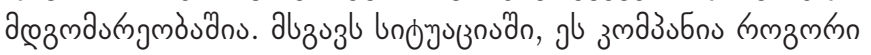

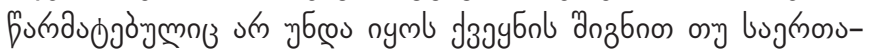

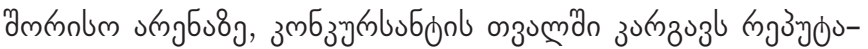

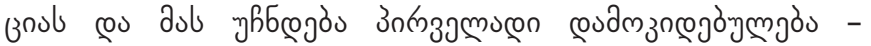

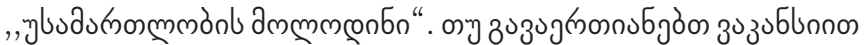

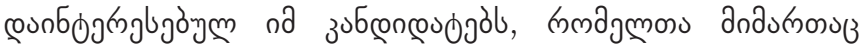

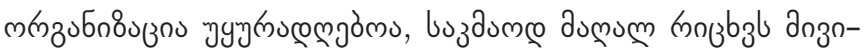

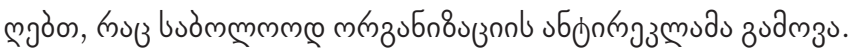

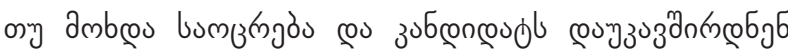

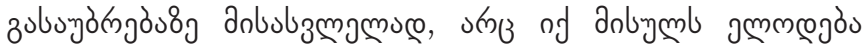

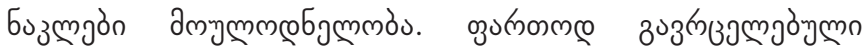

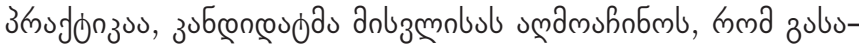

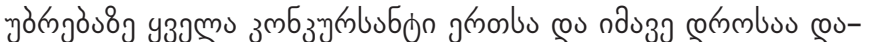

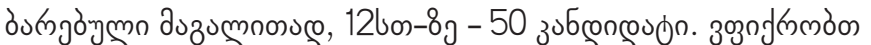

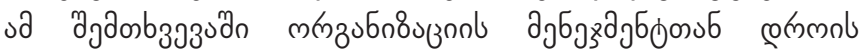

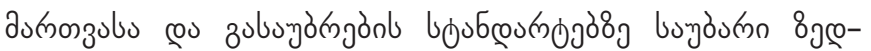

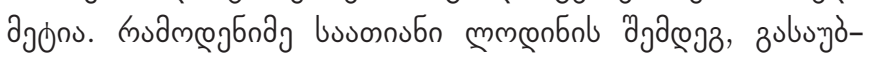

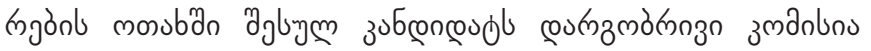

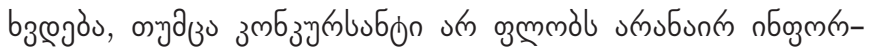

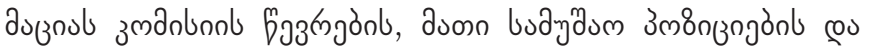

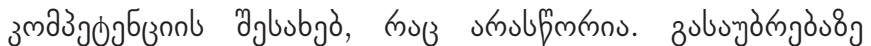

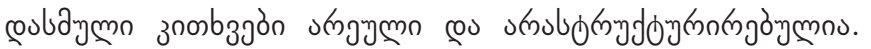

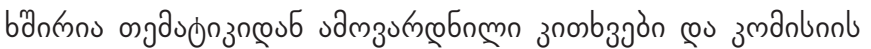

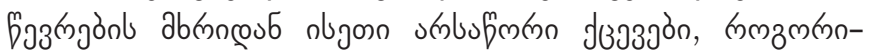

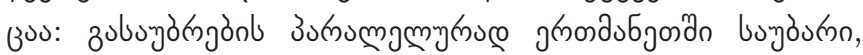

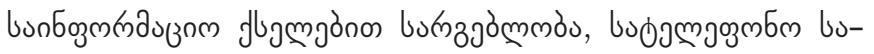

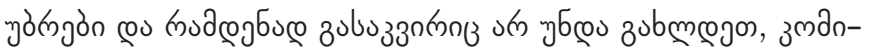

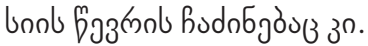

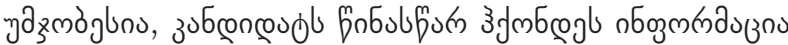

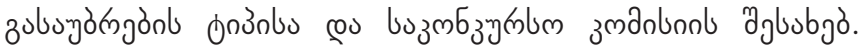

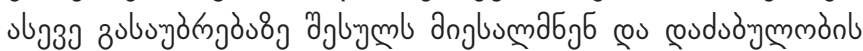

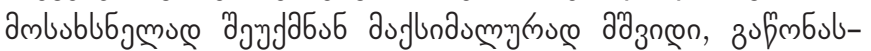

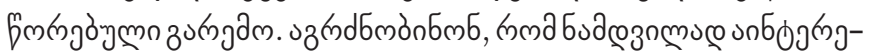

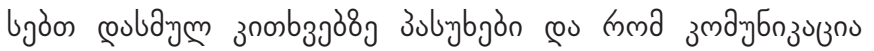
m nabbrnozos.

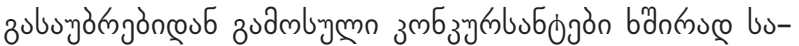

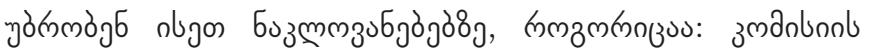

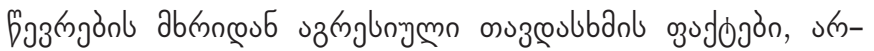

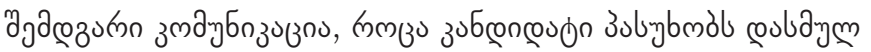

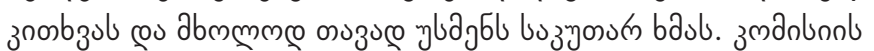

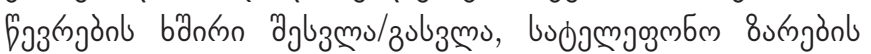

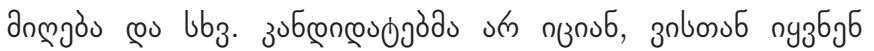
zulvugd

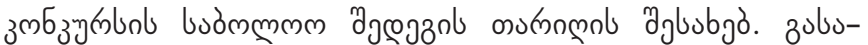

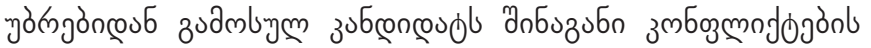

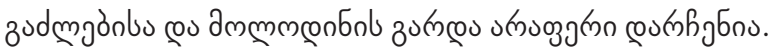

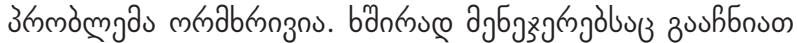
змбзचु

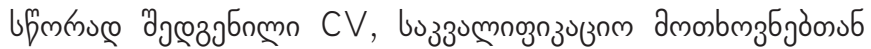

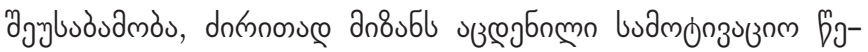

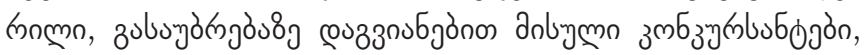

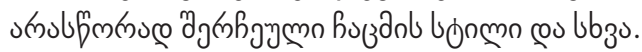

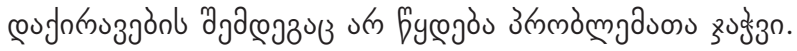

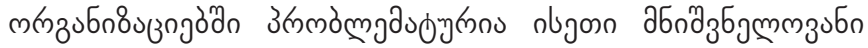

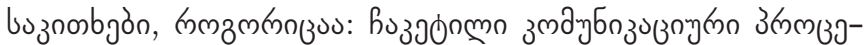

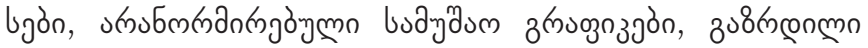

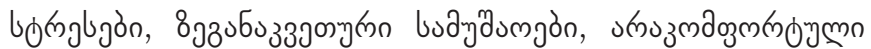

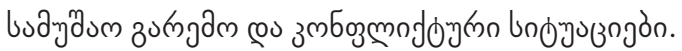

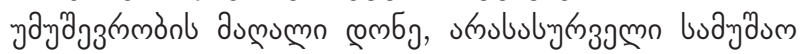

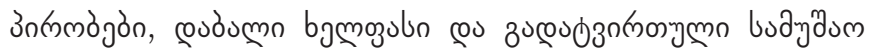
z

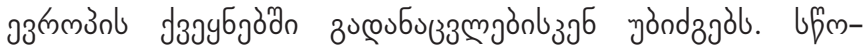

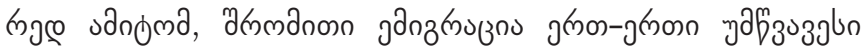
उ mmàmgasu bufurnos zammlonzol.

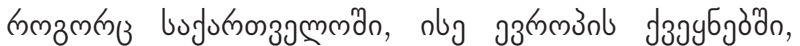

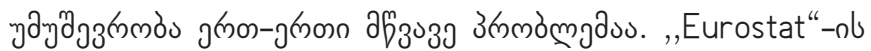

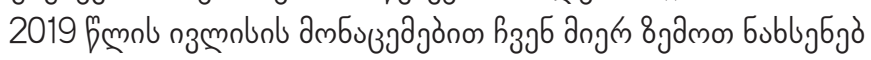

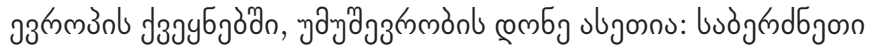

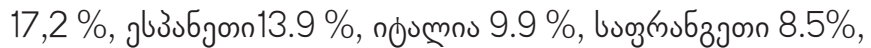

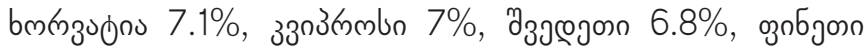

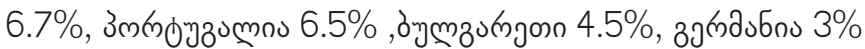
cou habjonn $2.1 \%$

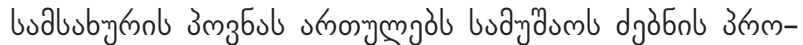

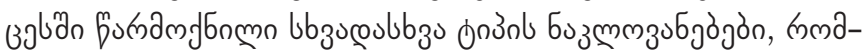

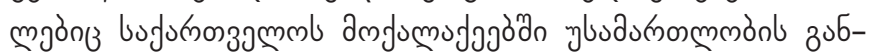

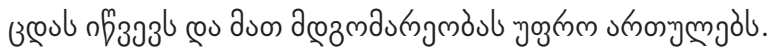

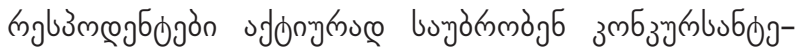

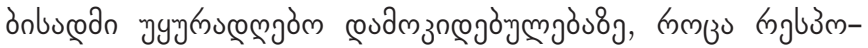

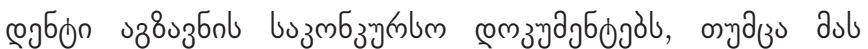

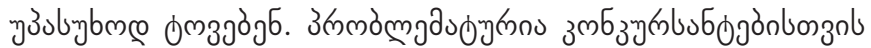

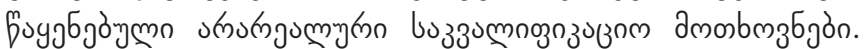

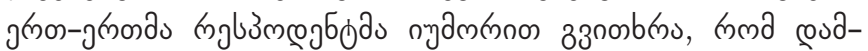

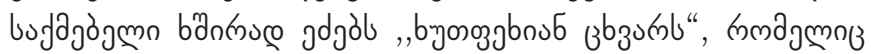

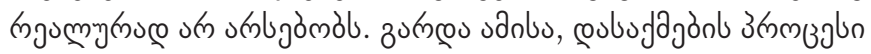

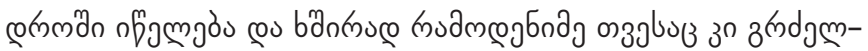
एоòu.

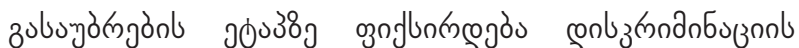

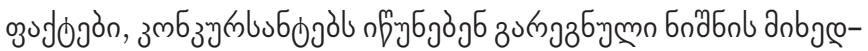

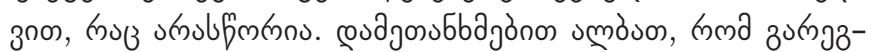

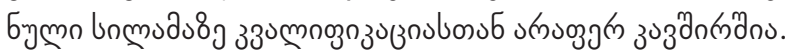

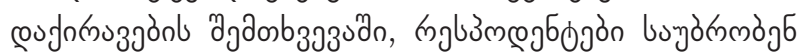

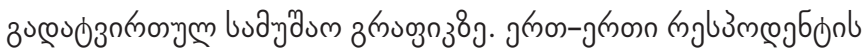

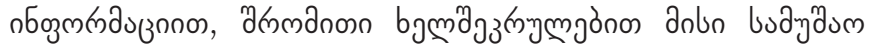

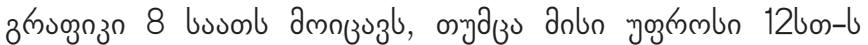

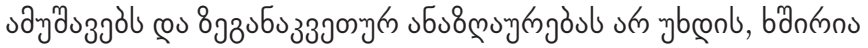

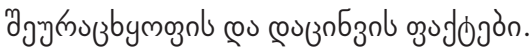




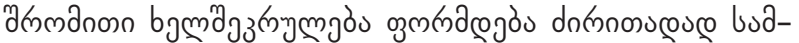

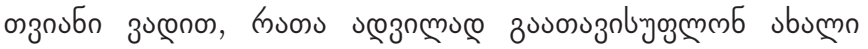

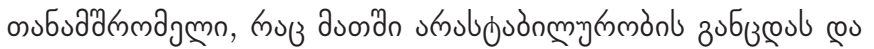
bong

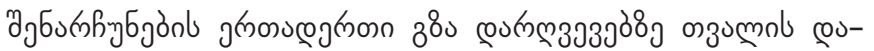

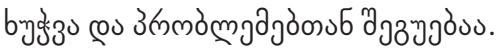

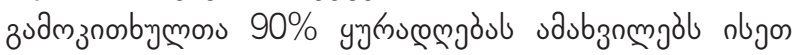

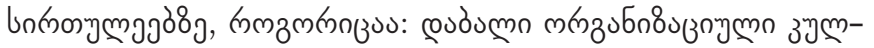

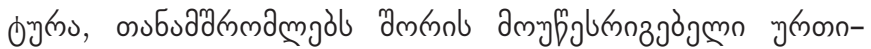

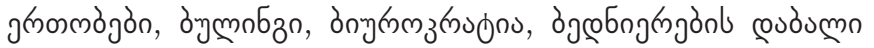

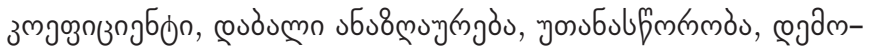

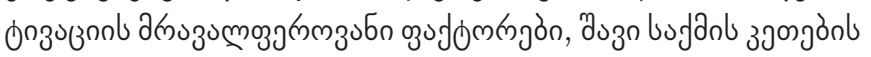

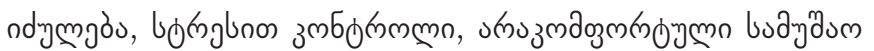

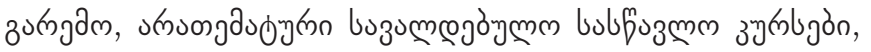

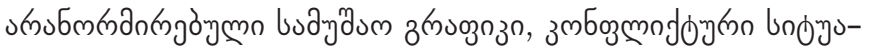

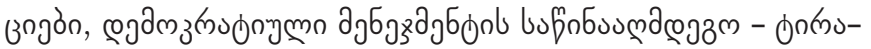

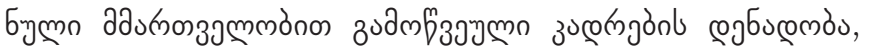

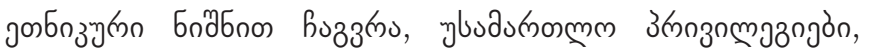

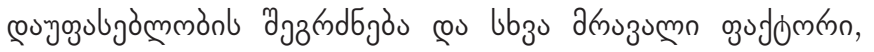

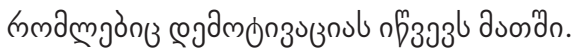

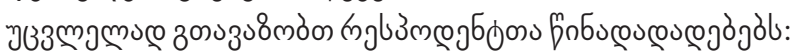

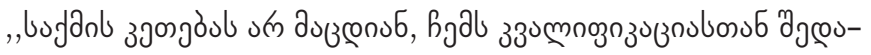

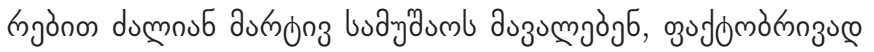

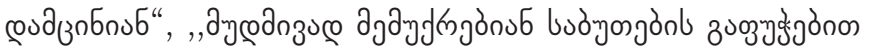

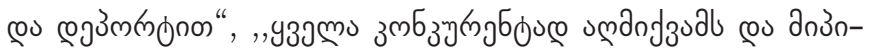

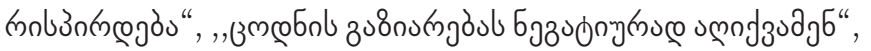

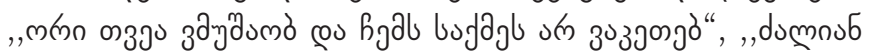

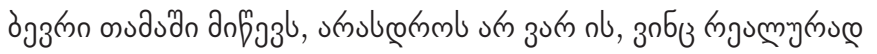

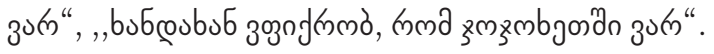

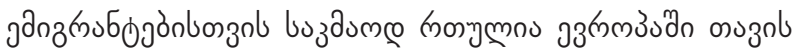

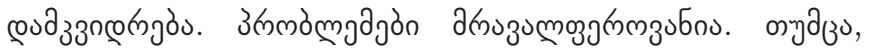

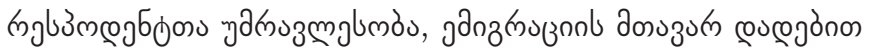

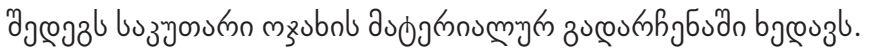

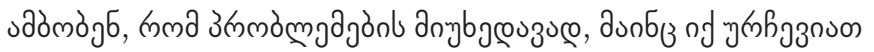
ymoुbs.

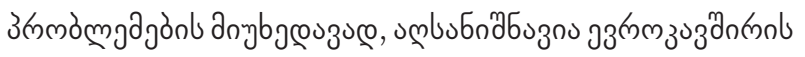
ذзаубара几

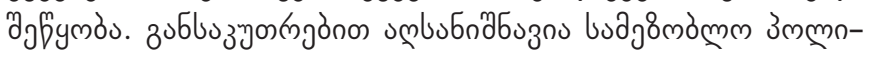

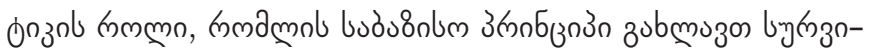

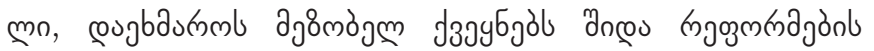

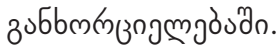

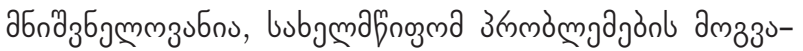

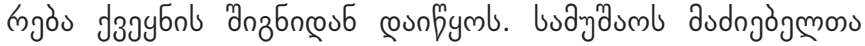

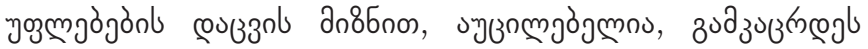

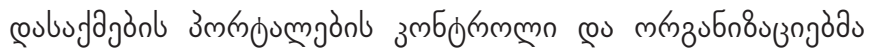

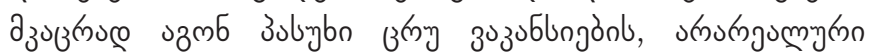

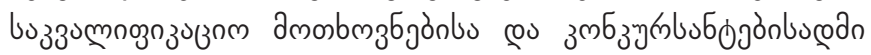

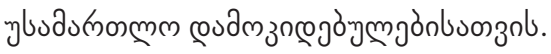

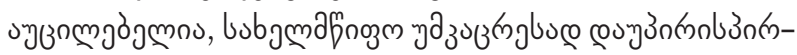
œ

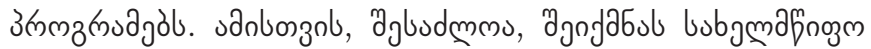

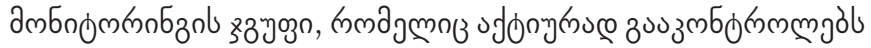

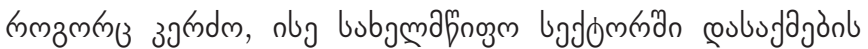

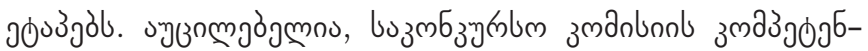

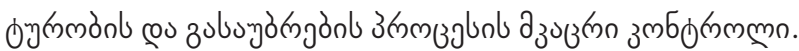

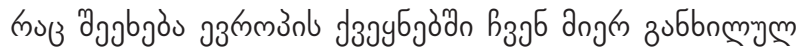

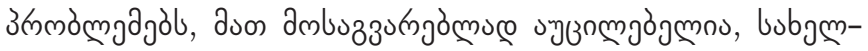

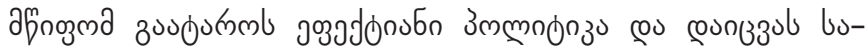

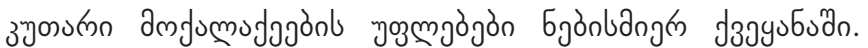

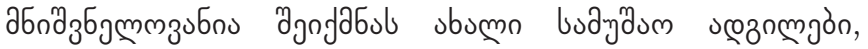

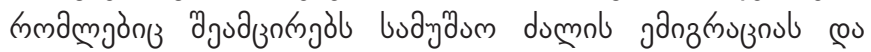

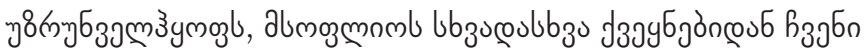

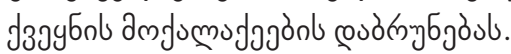

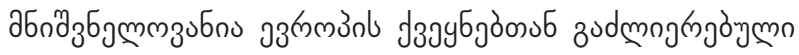

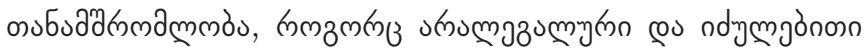

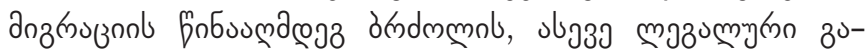

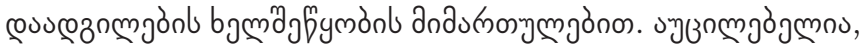

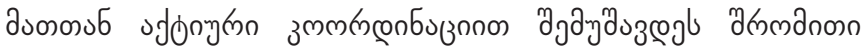

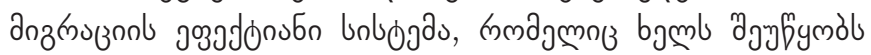

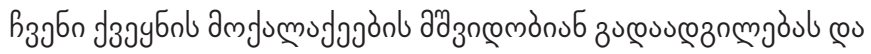
coubujajaul.

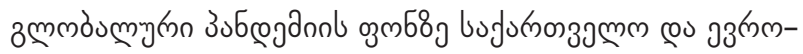

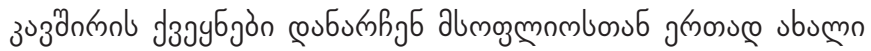
зодm

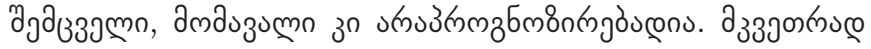

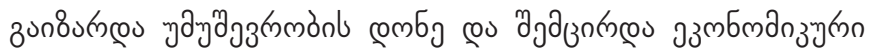

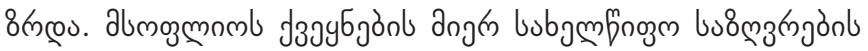

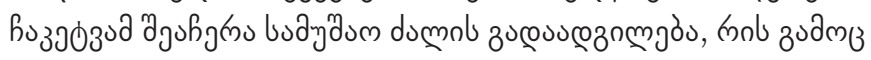

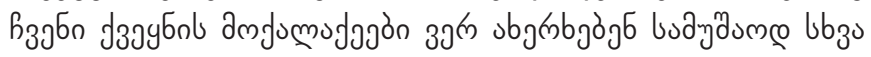

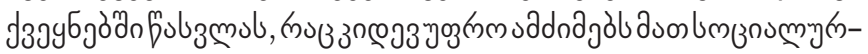

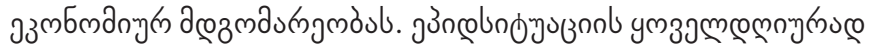

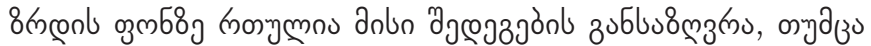

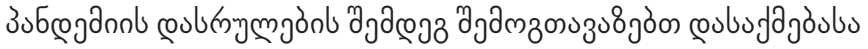

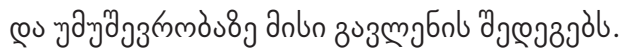




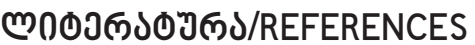

Brandolini.,( 2018) Andrea Measuring employment and unemployment. Bank of Italy, Italy.

Card. D., Kluve J. and Weber., A. (2015) „What Works? A meta analysis of recent active labor market program evaluations, National Bureau of Economic Research.

Knotek, Edward S (2007). How useful is Okun's Law. Economic Review, Federal Reserve Bank of Kansas City.

Messmer M., (2000) Job Hunting for Dummies, 2nd Edition USA.

Shearer, C.; Vey, J.S.; Kim, J.(2019) Where jobs are concentrating and why it matters to cities and regions (Washington, DC, Brookings Institution). Available at: https://www.brookings. edu/research/where-jobs-are-concentrating-why-itmattersto-cities-and-regions/ [20 Dec. 2019].

National Statistics Office of Georgia. External Trade and FDI. Retrieved from http://geostat.ge/index.php?action=page\&p_ id=134\&lang=eng on 2011-09-21

National Statistics Office of Georgia. Labor Force Statistics. Retrieved from http://geostat.ge/cms/site_images/_fi les/english/ methodology/labour\%20force\%20statistics\%20 Eng.pdf on 2011-08-01

Canadian Internationl Labour Network. Self-Employment in Transitional Economies: Entrepreneurship or Disguised Unemployment? Retrieved from http://www.ciln.mcmaster.ca/papers/seconf/transecns.pdf on 2011-09-07

I want to work, Employment Services 2020 Report. https://docs.employment.gov.au/system/files/doc/other/final_-_i_want_ to_work.pdf

https://www.geostat.ge/ka

https://ec.europa.eu/info/departments/eurostat-european-statistics_en 